\title{
Vitamin E is ineffective for symptomatic relief of knee osteoarthritis: a six month double blind, randomised, placebo controlled study
}

\author{
C Brand, J Snaddon, M Bailey, F Cicuttini
}

\begin{abstract}
Objective-There is a putative role for antioxidant treatment in osteoarthritis (OA) based on animal, epidemiological, and human clinical studies. Vitamin E, a fat soluble vitamin, is one of the major dietary antioxidants. Short term clinical studies using vitamin $E$ in the form of $\alpha$-tocopherol suggested a benefit over placebo of similar dimension to that of diclofenac for relief of $O A$ pain.

Methods-A six month, double blind, randomised, placebo controlled study of vitamin E 500 IU/day was carried out. Primary outcome measures were pain, stiffness, and function. Statistical analysis was performed on an intention to treat basis.
\end{abstract}

Results -77 patients were included in the study. Vitamin E showed no benefit over placebo at one month, three months, or six months for any of the outcome measures. The placebo group had higher pain levels $(p=0.15)$ and body mass index $(p=0.03)$ at baseline, and lower pain levels $(p=0.02)$ at completion of the study. Radiological score, exercise score, age, or antioxidant intake at baseline or six months did not differ between the groups. The reasons for the better performance of the placebo group are uncertain but may relate to the initially higher pain score and subsequent regression to the mean.

Conclusions-Vitamin E shows no benefit for the management of symptomatic knee $O A$. The role of vitamin $E$ in preventing OA progression is currently under investigation.

(Ann Rheum Dis 2001;60:946-949)

Department of Rheumatology, Alfred Hospital, Prahran, Vic, Australia

C Brand

Department of Epidemiology and Preventive Medicine, Monash University Medical School, Alfred Hospital, Prahran, Vic, Australia

J Snaddon

M Bailey

F Cicuttini

Correspondence to: Dr Brand

hollandc@ocean.com.au

Accepted 8 March 2000
Osteoarthritis (OA) is the most common arthritic condition affecting an increasingly aging population. ${ }^{1} \quad$ Non-steroidal antiinflammatory drugs (NSAIDs) have been the main treatment of OA for patients when simple analgesia is inadequate for symptomatic control. However, NSAIDs are associated with significant morbidity and mortality in the older population. ${ }^{2}$ Some drugs in this class have been reported to have deleterious effects on cartilage metabolism. ${ }^{3}$ Despite the recent introduction of cyclo-oxygenase-2 (COX-2) inhibitors, with a reduced toxicity profile, ${ }^{4}$ alternative treatments for symptoms in OA are still needed.

Recently, there has been increasing evidence that vitamin $\mathrm{E}$, a fat soluble vitamin, and one of the major dietary antioxidants, may have a role in OA. The mechanism of efficacy of vitamin $\mathrm{E}$ in $\mathrm{OA}$ is unknown, but, in the form of $\alpha$-tocopherol, it protects critical cellular structures against damage from oxygen free radicals and reactive products of lipid peroxidation. ${ }^{5}$ Animal, epidemiological, and human clinical studies have supported a role for antioxidant treatment in the management of OA knee. Incubation of rabbit articular cartilage in the presence of $\alpha$-tocopherol has been shown to preserve sustained load carrying capacity and viability. ${ }^{6}$ Kowsari et al using a three day food frequency questionnaire in 24 patients with rheumatoid arthritis (RA) and 12 with OA found both groups had dietary intakes of vitamin $\mathrm{E}$ that were below recommended dietary allowances. $^{7}$ When 640 participants in the Framington Osteoarthritis cohort Study ${ }^{8}$ were examined, results of a food frequency questionnaire suggested that high intake of antioxidants reduced the risk of cartilage loss and disease progression, though there was no association with incident OA. Several clinical studies have found therapeutic benefits of $\alpha$-tocopherol in the symptomatic treatment of $\mathrm{RA}^{9}{ }^{10}$ and OA. ${ }^{11-13}$ Short term clinical trials with a small number of patients have suggested that vitamin E treatment may be more effective than placebo in relieving pain ${ }^{11}$ and may have similar efficacy to diclofenac. ${ }^{13}$

To determine the clinical usefulness of vitamin $\mathrm{E}$ in the management of symptomatic knee OA, we performed a six month, double blind, randomised, placebo controlled trial of 500 IU vitamin $\mathrm{E}$.

\section{Methods}

Patients were recruited by a combined strategy, including advertising through local newspapers and the Victorian branch of the Arthritis Foundation of Australia as well as by collaboration with general practitioners, specialist rheumatologists, and orthopaedic surgeons. The study was approved by the ethics committee of the Alfred and Caulfield hospitals in Melbourne, Australia. All patients gave informed consent.

INCLUSION AND EXCLUSION CRITERIA

Men and women aged 40 years or more who fulfilled American Rheumatism Association clinical diagnostic criteria $^{14}$ for knee OA and had radiographic evidence of osteophytes or joint space narrowing were included. Patients were required to have pain on more than half the days of a month and at least one pain dimension of the Western Ontario and McMaster University Osteoarthritis Index $\left(\right.$ WOMAC $^{15}$ ) pain score above $20 \%$ using a 5 
$\mathrm{cm}$ visual analogue scale (VAS). In addition, a categorical measure of pain severity of at least mild (defined as no compromises of daily activities, frequent but tolerable pain that is worsened by unusual activity, and may take a pain reliever occasionally) was a prerequisite for study entry.

Patients with any known sensitivity to vitamin $\mathrm{E}$, receiving current anticoagulation treatment, with a previous stroke or history of poorly controlled hypertension were not eligible for the trial. Similarly, other major morbidities, such as cancer or life threatening illnesses, or inability to cooperate with study requirements and give informed consent, such as dementia, precluded entry. Patients awaiting knee replacement or in whom there was radiological evidence of grade IV knee $\mathrm{OA}^{16}$ were excluded from the study.

Patients already receiving anti-inflammatory drugs were not excluded if the dosage and regularity of administration was not expected to alter during the six month trial period. Analgesic and anti-inflammatory use was monitored during the study. Patients who had previously taken vitamin E supplements were not excluded from the study if assurance was given that no supplements would be taken during the period of the study. Vitamin E supplements were stopped at least 48 hours before entry.

TREATMENT PROGRAMME AND DATA COLLECTION Participants were randomly allocated in a double blind manner in equal numbers to receive either vitamin E 500 IU daily or placebo using a computer generated block randomisation program. Subjects randomly allocated to receive placebo were given soybean tablets that were identical in appearance to the vitamin $\mathrm{E}$ and were also taken once daily. Medication was given to participants at the baseline and three month visits. Compliance was assessed by returned pill counts at three and six months.

\section{OUTCOME MEASURES}

The primary outcome measures were pain, stiffness, and function dimensions as derived from WOMAC. Patients were asked to rate the change in these dimensions since their last visit on a $5 \mathrm{~cm}$ VAS. The incidence of pain in each knee in the month before review was assessed (less than 10 days, 10-15 days, more than half the month, every day). A categorical measure of pain severity for the 24 hours before review was documented (none, slight, mild, moderate, severe, extreme). Observer global assessment of current severity was also documented. Secondary outcome measures included analgesic and NSAID usage $(0=$ never used; $1=$ rarely used; 2 = used a few days/week; $3=$ used most days/week; 4 = used daily).

Subjects completed a questionnaire that included demographic data and current physical activity. ${ }^{17}$ Weight was measured to the nearest $0.1 \mathrm{~kg}$ (shoes, socks, and bulky clothing removed) with a single pair of electronic scales. Height was measured to the nearest $0.1 \mathrm{~cm}$ (shoes and socks removed) with a stadiometer. Body mass index (BMI; weight/height ${ }^{2}$ in $\mathrm{kg} / \mathrm{m}^{2}$ ) was calculated. Dietary intake of vitamin E, using a food questionnaire, ${ }^{18}$ was completed by patients at the start and end of the study.

Radiography before inclusion into the study included a weightbearing anteroposterior tibiofemoral view in full extension and skyline patella view. The blinded radiographs were read on two separate occasions by one investigator at completion of the study. Radiographic scoring of tibiofemoral OA and patellofemoral OA was made using a standardised radiographic atlas. ${ }^{17}$ The intraobserver reliability ranged between 0.87 and 0.92 for joint space narrowing and osteophytes at the tibiofemoral and patellofemoral joints.

\section{STATISTICAL ANALYSIS}

Primary analysis was performed on an intention to treat basis. Baseline characteristics were compared using the two sample $t$ test. Categorical variables were compared at baseline using the $\chi^{2}$ test for equal proportions. The mean differences at six months were assessed with paired $t$ tests, and adjustments for baseline differences made using covariate analysis. Results from the multivariate model are presented as adjusted means, with the adjustment made according to the method of least squares. Repeated measurement analysis was used to compare differences between vitamin $\mathrm{E}$ and placebo at each visit. With 38 patients in each arm, the study had an $80 \%$ power to detect a 30\% improvement in each of the three dimensions, where mean/SD in the whole population at baseline were pain (87.9/39.2), stiffness (43.4/21.7), and function (329.6/ 173.5)

\section{Results}

Four hundred patients underwent telephone screening, of whom 158 were interviewed. Seventy seven patients (45 female, 32 male) fulfilled study inclusion criteria and were randomly allocated to one arm of the study. Seventy two patients completed the study. Five withdrew before study completion (three owing to treatment failure, one relocated unexpectedly overseas, one developed osteonecrosis). Compliance was not significantly different between the groups (vitamin E 96.8 (SE 1.4) $v$ placebo 93.6 (SE 3.0), $\mathrm{p}=0.3$ ).

Table 1 presents baseline characteristics of all patients entered into the study. There were no significant baseline differences between the vitamin $\mathrm{E}$ and placebo groups except for BMI, which was higher in the placebo group (30.5 $\mathrm{v}$ 27.6, $\mathrm{p}=0.03$ ). Pain levels were also higher in this group, but the difference was not significant $(p=0.15)$. One patient in each group was taking vitamin $\mathrm{E}$ supplementation before study entry. Fourteen patients in each group were receiving NSAID treatment when they entered the study. Two patients receiving vitamin $\mathrm{E}$ and two receiving placebo stopped their NSAID during the six month study, and one further patient receiving vitamin E started an NSAID during this time. Mean differences in dietary vitamin $\mathrm{E}$ at six months did not differ significantly (mean difference vitamin E -1.6 
Table 1 Pretreatment characteristics of 77 patients

\begin{tabular}{lccl}
\hline & Vitamin E (SE) & Placebo (SE) & Difference (p value) \\
\hline Proportion female (\%) & 60 & 57 & $0.83\left(\chi^{2}\right)$ \\
Australian born (\%) & 76 & 54 & $0.04\left(\chi^{2}\right)$ \\
Mean age & $67.1(1.4)$ & $66.1(1.5)$ & 0.62 \\
BMI $^{\star}$ & $27.6(0.8)$ & $30.5(1.0)$ & 0.03 \\
Dietary vitamin E & $4.7(0.6)$ & $4.2(0.3)$ & 0.45 \\
Mean activity score & $6.0(0.2)$ & $6.2(0.2)$ & 0.61 \\
Mean pain score & $76.4(5.6)$ & $87.7(5.5)$ & 0.15 \\
Mean stiffness score & $38.8(3.0)$ & $41.5(2.9)$ & 0.51 \\
Mean physical function score & $303.6(21.4)$ & $325.8(21.1)$ & 0.46 \\
Mean pain frequency & $3.1(0.18)$ & $3.2(0.18)$ & 0.91 \\
Categorical pain score & $2.0(0.13)$ & $2.3(0.16)$ & 0.14 \\
Observer global score & $1.9(0.12)$ & $2.2(0.14)$ & 0.38 \\
Radiological score (SE) & $4.9(0.5)$ & $4.5(0.5)$ & 0.57 \\
NSAID ${ }^{\star}$ frequency & $1.5(1.8)$ & $1.5(1.9)$ & 0.96 \\
Analgesic frequency & $1.2(1.4)$ & $1.2(1.5)$ & 0.87 \\
\hline
\end{tabular}

${ }^{\star} \mathrm{BMI}=$ body mass index; NSAID = non-steroidal anti-inflammatory drug.

Table 2 Repeated measurement analysis of vitamin $E$ versus placebo over six months. (adjusted means)

\begin{tabular}{|c|c|c|c|c|c|}
\hline & Baseline & 1 Month & 3 Months & 6 Months & $\begin{array}{l}\text { Change between } \\
\text { groups over time } \\
\text { (p value) } \ddagger\end{array}$ \\
\hline \multicolumn{6}{|l|}{ Paint } \\
\hline Vitamin E & 49.23 & 82.84 & 67.23 & 71.54 & \\
\hline Placebo & 61.67 & 83.61 & 79.39 & 66.70 & \\
\hline p Value & $0.09 \dagger$ & 0.93 & 0.17 & 0.59 & 0.24 \\
\hline \multicolumn{6}{|l|}{ Stiffnesst } \\
\hline Vitamin E & 25.70 & 41.77 & 34.47 & 34.14 & \\
\hline Placebo & 28.75 & 39.58 & 35.23 & 33.63 & \\
\hline p Value & 0.45 & 0.66 & 0.88 & 0.92 & 0.43 \\
\hline \multicolumn{6}{|l|}{ Function $†$} \\
\hline Vitamin E & 209.44 & 304.70 & 258.08 & 279.69 & \\
\hline Placebo & 241.88 & 320.54 & 305.07 & 279.02 & \\
\hline p Value $e^{\star}$ & 0.24 & 0.63 & 0.16 & 0.99 & 0.44 \\
\hline \multicolumn{6}{|c|}{ Pain frequency } \\
\hline Vitamin E & 2.92 & 2.72 & 2.47 & 2.08 & \\
\hline Placebo & 2.92 & 2.82 & 2.67 & 2.34 & \\
\hline $\mathrm{p}$ Value & 0.99 & 0.74 & 0.48 & 0.39 & 0.72 \\
\hline \multicolumn{6}{|c|}{ Categorical pain } \\
\hline Vitamin E & 1.83 & 1.86 & 1.66 & 1.82 & \\
\hline Placebo & 2.12 & 1.90 & 1.86 & 1.47 & \\
\hline $\mathrm{p}$ Value & 0.18 & 0.87 & 0.35 & 0.12 & 0.12 \\
\hline \multicolumn{6}{|c|}{ Observer global assessment } \\
\hline Vitamin E & 1.84 & 1.63 & 1.51 & 1.66 & \\
\hline Placebo & 1.99 & 1.72 & 1.74 & 1.45 & \\
\hline $\mathrm{p}$ Value & 0.46 & 0.63 & 0.22 & 0.30 & 0.32 \\
\hline
\end{tabular}

${ }^{\star}$ Significance in differences between least squares means.

†All values adjusted for sex, ethnicity, age, antioxidant intake, arthritis score, exercise level, and body mass index.

$\ddagger$ The significance of changes over time was assessed by a visit by group interaction.

$(\mathrm{SE} 0.5) v$ mean difference placebo -1.0 (0.5), $\mathrm{p}=0.41)$.

Neither group showed a significant improvement in pain, stiffness, and physical function over six months. The placebo group had a smaller increase in pain score between baseline and six months than the vitamin $\mathrm{E}$ group $(p=0.02)$ after adjusting for age, sex, BMI, radiological OA severity, analgesic and antiinflammatory drug use. Repeated measurement analysis of outcome measures at each visit showed no significant differences in any dimension (primary or secondary outcomes) at any time point (table 2). Although there were differences within groups between visits, there was no difference between groups over time (table 2).

\section{Discussion}

Our results do not support a role for vitamin E in the treatment of symptoms in knee OA. We showed no benefit of vitamin $\mathrm{E}$ in any measure of pain score, nor in stiffness or function. Observer assessment demonstrated no significant differences. Analgesia or NSAID usage, surrogate markers of clinical efficacy, did not change significantly between those taking vitamin E compared with those receiving placebo. At six months, the deterioration in pain score was less in the placebo group than in the vitamin $\mathrm{E}$ group after adjusting for age, sex, BMI, radiological score, analgesic and antiinflammatory drug use. When the earlier time points, one month and three months, were examined, again no significant benefit of vitamin E compared with placebo was seen.

Our results differ from the study of Machtey and Ouaknine. ${ }^{11}$ Those authors found a $52 \%$ improvement in the group treated with vitamin E compared with $4 \%$ in the placebo group. However, they studied only 32 patients. OA was present at any site and subjects were randomly allocated into a short term (10 day crossover) study comparing vitamin E 600 $\mathrm{mg} /$ day with placebo. The administrators of the treatment were not blinded. The outcome measures included a simple daily patient recorded global improvement scale and frequency of analgesic intake. The small numbers, heterogeneous site of OA, short duration of treatment, and lack of double blinding limit interpretation of these results. A pilot study of vitamin E efficacy in 50 patients with $\mathrm{OA}^{12}$ (site not specified in the abstract) in a double blind, placebo controlled trial of over six weeks' duration found that vitamin $\mathrm{E}$ (400I.E) was better than placebo for pain relief and reducing the frequency of analgesic treatment. A further three week randomised, double blind, comparative study of vitamin $\mathrm{E}^{13}$ (400 mg/day) versus diclofenac ( $50 \mathrm{mg}$ three times daily) in 34 patients with hip OA or 19 with knee OA showed that the two agents had equal efficacy. Given the small numbers of patients with $\mathrm{OA}$ at each site, this study may have been underpowered to detect a difference in treatment efficacy between the two groups. Our study showed no significant difference even when we examined earlier time points of one and two months.

The reason why placebo performed better than vitamin $\mathrm{E}$ is unclear. The only significant baseline difference between the two groups was BMI, which one could postulate might be associated with earlier and more severe OA. However, a final analysis allowed adjustment for this variable. In addition, there was no difference in radiological OA score between the two groups at baseline, and no differences at baseline or at six months in the frequency of use of analgesic and anti-inflammatory drugs. Possibly, although we adjusted for baseline pain score, this still, in part, represents regression to the mean because the placebo group had more significant pain at the start of the study than the vitamin E group.

Overall drug compliance in the study participants was very high and dietary intake assessment of antioxidants showed no significant difference between the two groups. As serum levels of vitamin $\mathrm{E}$ were not determined we cannot exclude the possibility that differences in antioxidant levels might have existed and influenced the final results. However, given the high dose of vitamin E supplementation, this is unlikely to be the case. In addition, because we only examined symptoms in this 
study, we cannot exclude the possibility that vitamin $\mathrm{E}$ affects progression of knee OA. No randomised controlled trial data exist to support this. This is currently mainly supported by observational data such as the Framingham Study, which has suggested a reduction in the prevalence, but not the incidence, of knee OA in those with a high dietary intake of vitamin $\mathrm{E}$.

No adverse affects were associated with vitamin $\mathrm{E}$ in our study. This is consistent with published reports, which suggest that short term vitamin $\mathrm{E}$ use is safe. ${ }^{20}$ However, there has been some doubt about its long term use after a report of an increase in cerebral haemorrhage among men taking vitamin $\mathrm{E}$ for $5-8$ years. ${ }^{21}$ Despite the widespread, community interest in the use of "natural" treatments in OA, our data do not support the use of vitamin $E$ for treatment of symptoms in knee OA, and emphasise the importance of testing such treatments in double blind randomised studies. Such caution is reinforced by the failure of randomised controlled trials to support observational studies suggesting a better cardiovascular prognosis in subjects with high antioxidant intake. ${ }^{22}$

The authors thank Associate-Professor PJ Ryan for his advice and support of this project.

1 Felson DT, Naimark A, Anderson JJ, Kazis L, Castelli W, Meenan RF, et al. The prevalence of knee osteoarthritis in the elderly: the Framingham Osteoarthritis Study. Arthritis Rheum 1987;30:914-18.

2 Griffin MR, Piper JM, Daugherty JR, Snowden M, Ray WA. Non-steroidal anti-inflammatory drug use and increased Med 1991;114:257-63.

3 David MJ, Vignon E, Peschard MJ, Broquet P, Louisot P, Richard M. Effect of non-steroidal anti-inflammatory drugs (NSAID) on glycosyltransferase activity from human osteoarthritic cartilage. Br J Rheumatol 1992;31(suppl osteoarthritic $13-17$.

4 Hawkey CJ. Cox-2 inhibitors. Lancet 1999;353:307-14.
5 Azzi A, Boscobonik D, Hensey C. The protein kinase C family. Eur J Biochem 1992;208:547-57.

6 Black J, Shadle CA, Parsons JR, Brighton CT. Articular cartilage preservation and storage. Arthritis Rheum 1979;22: 1102-8.

7 Kowsari B, Finnie SK, Carter R, Love J, Katz P, Longley S, et al. Assessment of the diet of patients with rheumatoid arthritis and osteoarthritis. J Am Diet Assoc 1983;82: 657-9.

8 McAlindon TE, Jacques P, Zhang Y, Hannan MT, Aliabadi $\mathrm{P}$, Wissman B, et al. Do antioxidant micronutrients protect against the development and progression of knee osteoarthritis? Arthritis Rheum 1996;39:648-56.

9 Edmonds SE, Winyard PG, Guo R, Kidd B, Merry P, Langrish-Smith A, et al. Putative analgesic activity of repeated oral doses of vitamin $\mathrm{E}$ in the treatment of repeated oral doses of vitamin $\mathrm{E}$ in the treatment of
rheumatoid arthritis. Results of a prospective placebo controlled double blind trial. Ann Rheum Dis 1997;56:64955

10 Wittenborg A, Petersen G, Lorkowski G, Brabant T. Effectiveness of vitamin $\mathrm{E}$ in comparison with diclofenac sodium in treatment of patients with chronic polyarthritis. Z Rheumatol 1998;57:215-21.

11 Machtey I, Ouaknine L. Tocopherol in osteoarthritis: a controlled pilot study. J Am Geriatr Soc 1978;26:328-30.

12 Blankenhorn $G$. Clinical effectiveness of vitamin $E$ in activated arthroses. A multi center placebo-controlled activated arthroses. A multi center placebo-co

13 Scherak O, Kolarz G, Schodl C, Blankenhorn G. High dosage vitamin $\mathrm{E}$ therapy in patients with activated arthrosis. $\mathrm{Z}$ Rheumatol 1990;49:369-73.

14 Altman R, Asch E, Bloch D, Bole G, Borenstein K, Brandt $\mathrm{K}$, et al. Development of criteria for classification and reporting of osteoarthritis. Arthritis Rheum 1986;29:103949

15 Bellamy N. Outcome measures in osteoarthritis clinical trials. J Rheumatol 1995;22(suppl 43):49-51.

16 Kellgren JA, Lawrence JS. Atlas of standard radiographs: epidemiology

17 Spector TD, Harris PA, Hart DJ, Cicuttini FM, Nandra D, Etherington J, et al. Risk of osteoarthritis associated with running: a radiological survey of female ex-athletes and running: a radiological survey of female ex-athletes an

18 McCarty CA, De Paola C, Livingston PM, Taylor HR. ReliMcCarty CA, De Paola C, Livingston PM, Taylor HR. Reli-
ability of a food frequency questionnaire to assess dietary antioxidant intake. Ophthalmic Epidemiol 1997;4:33-9.

19 Spector T, Cooper C, Cushnaghan J, Hart DJ, Dieppe P. $A$ radiographic atlas of knee osteoarthritis. London: Springer, 1992:1-15.

20 Meyers DG, Maloley PA, Weeks D. Safety of antioxidant vitamins. Arch Intern Med 1996;156:925-35.

21 The Alpha-Tocopherol, Beta Carotene Cancer Prevention Study Group. The effect of vitamin E and beta carotene on the incidence of lung cancer and other cancers in male smokers. N Engl J Med 1994;330:1029-35.

22 Scheen AJ. Antioxidant vitamins in the prevention of cardiovascular diseases. 2nd part: result of clinical trials. Rev Med Liege 2000;55:105-9. 